\title{
Clinical Phenotype and Contagiousness of Early Breakthrough SARS-CoV-2 Infections after BNT162b2 COVID-19 mRNA Vaccine: A Parallel Cohort Study in Healthcare Workers
}

\author{
Mattia Trunfio ${ }^{1, *(\mathbb{D})}$, Federica Verga ${ }^{2}$, Valeria Ghisetti $\left.{ }^{3} \mathbb{(}\right)$, Elisa Burdino ${ }^{3}$, Teresa Emanuele ${ }^{2}$, Stefano Bonora ${ }^{1}$, \\ Giovanni Di Perri ${ }^{1}$ and Andrea Calcagno ${ }^{1}$ (D) \\ 1 Unit of Infectious Diseases, Department of Medical Sciences, Amedeo di Savoia Hospital, University of \\ Torino, Corso Svizzera 164, 10159 Torino, Italy; stefano.bonora@unito.it (S.B.); \\ giovanni.diperri@unito.it (G.D.P.); andrea.calcagno@unito.it (A.C.) \\ 2 Occupational Medicine Unit, Maria Vittoria Hospital, 10159 Torino, Italy; \\ federicacristina.verga@aslcittaditorino.it (F.V.); teresa.emanuele@aslcittaditorino.it (T.E.) \\ 3 Microbiology and Molecular Biology Unit, Amedeo di Savoia Hospital, 10159 Torino, Italy; \\ valeria.ghisetti@gmail.com (V.G.); elisa.burdino@libero.it (E.B.) \\ * Correspondence: mattia.trunfio@edu.unito.it; Tel.: +39-011-439-3884
}

Citation: Trunfio, M.; Verga, F.; Ghisetti, V.; Burdino, E.; Emanuele, T.; Bonora, S.; Di Perri, G.; Calcagno, A. Clinical Phenotype and Contagiousness of Early Breakthrough SARS-CoV-2 Infections after BNT162b2 COVID-19 mRNA Vaccine: A Parallel Cohort Study in Healthcare Workers. Vaccines 2021, 9 , 1377. https://doi.org/10.3390/ vaccines 9121377

Academic Editors: Soo-Hong Lee,

Hansoo Park, Jagathesh

Chandra Rajendran and

K. S. Jaganathan

Received: 4 November 2021

Accepted: 20 November 2021

Published: 23 November 2021

Publisher's Note: MDPI stays neutral with regard to jurisdictional claims in published maps and institutional affiliations.

Copyright: () 2021 by the authors. Licensee MDPI, Basel, Switzerland. This article is an open access article distributed under the terms and conditions of the Creative Commons Attribution (CC BY) license (https:// creativecommons.org/licenses/by/ $4.0 /)$.

\begin{abstract}
We evaluated the clinical protection of BNT162b2 mRNA vaccine in healthcare workers (HCWs) and how COVID-19 manifestations and contagiousness change as the time since first dose increases. A matched (1:2 ratio) parallel cohort study was performed. During the first three months of vaccination campaign, HCWs of the entire health district ASL Città di Torino (Turin, Italy) were classified according to SARS-CoV-2-positivity in respect of the vaccination schedule: post-first-dose (fHCWs, $<12$ days), partially (PHCWs, $\geq 12$ from first dose to $\leq 7$ days after the second), and totally vaccinated (THCWs, $\geq 8$ days after the second dose). Age-/sex-matched unvaccinated controls were randomly selected from all the SARS-CoV-2-positivity detected in the same district and period. Previous infections were excluded. Clinical and virologic data (ORF1ab gene cycle threshold values, $\mathrm{Ct}$ ) were recorded. In total, $6800 \mathrm{HCWs}$ received at least one dose, and 55 tested positive subsequently: $20 \mathrm{fHCWs}, 25$ PHCWs, 10 THCWs. Furthermore, 21.8\% of breakthrough infections were in male, with a median age of 49 years (32-56), and 51.4\% occurred while SARS-CoV-2 B.1.1.7 variant was predominant. The incident relative risk was $0.13(0.12-0.15)$ for PHCWs and $0.06(0.05-0.07)$ for THCWs. Compared to controls $(n=110)$, no difference was observed in fHCWs, while PHCWs and THCWs showed higher prevalence of asymptomatic infections, fewer signs/symptoms with a milder systemic involvement, and significantly higher Ct values (PHCWs 30.3 (24.1-35.5) vs. 22.3 (19.6-30.6), $p=0.023$; THCWs 35.0 (31.3-35.9) vs. 22.5 (18.2-30.6), $p=0.024)$. Duration of symptoms was also shorter in THCWs (5 days (3-6) vs. 9 (7-14), $p=0.028$ ). A linear increase of 3.81 points in $\mathrm{Ct}$ values was observed across the groups by vaccination status $(p=0.001)$ after adjusting for age, sex, comorbidities, and time between COVID-19 onset and swab collection. BNT162b2 decreased the risk of PCR-confirmed infections and severe disease, and was associated with a virologic picture of lesser epidemiologic concern as soon as 12 days after the first vaccine dose.
\end{abstract}

Keywords: COVID-19; vaccine; contagiousness; cycle threshold; symptoms; protection; clinical manifestations; mRNA vaccines; BNT162b2

\section{Introduction}

Safety and efficacy of BNT162b2 COVID-19 mRNA vaccination (BioNTech-Pfizer) have been reported in both the registration phase III trial and early phase IV follow-up, showing a 95\% efficacy against symptomatic SARS-CoV-2 infections starting as soon as seven days after the second dose [1-3]. Despite the undoubted efficacy of anti-SARS-CoV-2 vaccines in reducing symptomatic infections, several questions remain unresolved. 
Several countries are extending the interval between vaccine doses to maximize initial nationwide coverage, but data on long-term effectiveness of modified administration schedules as well as early effectiveness of first doses are lacking. The first real-word observations suggest variable effectiveness (42-85\%) against SARS-CoV-2 after first BNT162b2 dose [4-6], but pooled analyses are hard to perform due to variability in settings, outcomes definition, and timing from doses.

As for post-vaccine symptomatic infections, the placebo-controlled phase III efficacy trial reported that no more than $10 \%$ of severe COVID- 19 cases with onset after the first dose occurred in BNT162b2 recipients [1]. While epidemiological data reporting rates of symptomatic and asymptomatic infections between vaccinated and unvaccinated subjects are emerging, a detailed characterization of the clinical and viro-immunological features of the disease in vaccinated subjects remains poor, mainly due to the rarity of the event $[7,8]$. Knowledge of the severity and infectiousness of cases in vaccinated individuals will inform the risk posed by healthcare workers (HCWs) to vulnerable patients and add to understanding of the transmission dynamics in highly vaccinated populations. Nevertheless, currently very little evidence is available on the plausible reduced contagiousness of vaccinated individuals $[9,10]$.

Herein, we have compared clinical and virological data of vaccinated HCWs later resulting SARS-CoV-2-positive and grouped according to the time elapsed between BNT162b2 administration and swab positivity with SARS-CoV-2-positive age-/sex-matched unvaccinated controls detected in the same health district and during the same period. We sought to assess whether and to what extent early clinical protection from first vaccine dose develops among HCWs and whether the clinical picture and the entity of transmission potential could change as the time since first vaccine dose increases.

\section{Materials and Methods}

We conducted a matched (1:2 ratio) case-control parallel cohort study on all the HCWs of the entire health district ASL Città di Torino (Turin, Italy) who tested SARS-CoV-2 positive at nasal-pharyngeal swab (NPs) after receiving BNT162b2 vaccination; the enrollment started from the beginning of the vaccination campaign (27 December 2020) until 1 April 2021, while HCWs underwent periodical molecular testing for SARS-CoV-2 infection regardless of their clinical status (every 2 weeks) and on the occurrence of compatible signs and/or symptoms.

SARS-CoV-2-positive HCWs were classified into three groups:

(A) Post-first dose HWCs (fHCWs), when infection was detected $<12$ days after the first vaccine dose;

(B) Partially vaccinated HCWs (PHCWs), when the positivity was detected from day 12 after the first dose to day 7 after the second dose;

(C) Totally vaccinated HCWs (THCWs), when SARS-CoV-2 positivity was detected $\geq 8$ days after the second dose.

The vaccination schedule was settled as per registration trial: at day 0 first BNT162b2 dose and at day 21 the second dose. BNT162b2 has proved to induce the production of neutralizing antibodies as soon as 10 days after the first dose, developing a partial protection as soon as 12 days after that [1,11-13]. Therefore, we adopted this temporal cutoff to discriminate between $\mathrm{fHCWs}$, which could also include infections contracted before the vaccine administration (considering SARS-CoV-2 incubation period) and PHCWs. The cut-off of $\geq 8$ days after the second dose was adopted to discriminate as soon as possible between partially and THCWs as BNT162b2 effectiveness against symptomatic infections has been reported $92 \%$ already $>7$ days after the second dose [3] and to assess potential early differences.

Cases were compared to age-/sex-matched unvaccinated controls (1:2 ratio). Controls were randomly selected (simple random sampling) from the sampling frame represented by all the SARS-CoV-2 positive NPs results detected in the same health district ("Le Chiuse" testing center, ASL Città di Torino, the main testing center for general population referring 
to the same health district of HCWs) from 27 December 2020 to 1 April 2021 to infer similar prevalence of circulating SARS-CoV-2 strains. Individuals with evidence of prior SARSCoV-2 infection (either due to PCR or antibody) were excluded from cases and controls.

Data were recorded by phone interviews following a structured questionnaire to limit recall and different examiner bias (demographics, presence and type of signs and symptoms, date of COVID-19 onset, immunocompromising conditions or medications, comorbidities, previous SARS-CoV-2 positivity, and SARS-CoV-2 positive contacts) and from occupational medicine and laboratory records (dates of vaccinations, date of first positive NPs, PCR cycle threshold values -Ct-, and previous SARS-CoV-2 positivity). The considered comorbidities were any cardiovascular/lung diseases, overweight/obesity, current or previous smoking, any solid or haematological cancer, and immunological/hematological disorders.

The NPs of both cases and controls were processed by the same Molecular Biology laboratory, (Amedeo di Savoia hospital, Turin) using Cobas ${ }^{\circledR}$ SARS-CoV-2 Test (Roche Molecular Systems, Branchburg, NJ, USA), a real-time RT-PCR test for the qualitative detection of the open reading frame $1 \mathrm{ab}$ (ORF1ab, a SARS-CoV-2-specific nonstructural region), and a conserved region of the structural protein envelope (E) gene for pan-sarbecovirus detection. Ct of ORF1ab only were considered to have a uniform inverse proxy of viral load. The ABI Prism 7500 thermal cycler (Thermo Fisher Scientific, Waltham, MA, USA) was used for PCR amplification.

The study was approved by the Inter-departments Ethics Committee A.O.U. Città della Salute e della Scienza, A.O. Ordine Mauriziano di Torino, and A.S.L. Città di Torino (protocol n.0065839-00304/2020).

Nonparametric tests were adopted for statistical analyses performed by SPSS v27 (IBM statistics). Deletion methods were applied for missing data. Categorical variables are presented as absolute value (proportion) while continuous variables as median (interquartile range). A single-step enter method linear regression was run to evaluate changes in $\mathrm{Ct}$ values at diagnosis according to vaccination status and other significant variables previously reported to affect early viral amount [14].

\section{Results}

During the study period, $5901 \mathrm{HCW}$ sere administered the entire vaccination schedule of BNT162b2, while the other 899 HCWs were administered with the first dose. No severe adverse reaction to the vaccination was reported; minor adverse effects after first dose were reported always within the first week and did not affect COVID-19 sign and symptoms detection for PHCWs; as for the second dose, three patients developed symptoms at day 3, 7, and 7 from the dose, but the clinical assessment and follow-up ruled out potential reactions to the vaccination in asymptomatic infections.

In total, $55 \mathrm{HCW}$ tested positive to SARS-CoV- 2 after vaccination: $41.8 \%, 50.9 \%$, and $7.3 \%$ were doctors, nurses, and other healthcare staff members, respectively. Twenty were positive at a median time of 5 days (3-8) after the first dose (fHCWs); 25 tested positive at a median time of 17 (15-21) days after the first dose (PHCWs); and 10 tested positive at a median time of 33 days (12-46; THCWs) after the second dose. None of the positive cases between the two doses received the second one; 7 cases belonging to the PHCWs group received the second dose as they tested positive within one week after that. Demographic and clinical characteristics of cases and controls are shown in Table 1 and Supplementary Table S1. 
Table 1. Comparison of demographic, clinical, and virological features of partially and totally vaccinated SARS-CoV-2-positive healthcare workers and corresponding age- and sex-matched unvaccinated controls.

\begin{tabular}{|c|c|c|c|}
\hline Parameter & $\begin{array}{l}\text { Partially Vaccinated } \\
\text { HCWs }(n=25)\end{array}$ & $\begin{array}{l}\text { Controls } \\
(n=50)\end{array}$ & $p$ \\
\hline Age, years & $54(40-58)$ & $54(40-58)$ & 1.00 \\
\hline Male, $n$ & $5(20.0 \%)$ & $10(20.0 \%)$ & 1.00 \\
\hline Caucasian, $n$ & $23(92.0 \%)$ & $47(94.0 \%)$ & 0.932 \\
\hline Comorbidities/subject, $n$ & $0(0-1)$ & $0(0-1)$ & 0.283 \\
\hline Asymptomatic infections, $n$ & $9(36.0 \%)$ & $1(2.0 \%)$ & $<0.0001$ \\
\hline $\begin{array}{c}\text { Signs and Symptoms, } n * \\
\text { Fever } \\
\text { Cough } \\
\text { Rhinorrhea } \\
\text { Pharyngitis } \\
\text { Dyspnea } \\
\text { O/G dysfunction } \\
\text { Headache } \\
\text { Arthromyalgia } \\
\text { Asthenia/Malaise } \\
\text { Nausea/Vomiting } \\
\text { Diarrhea }\end{array}$ & $\begin{array}{l}3(18.7 \%) \\
6(37.5 \%) \\
5(31.2 \%) \\
3(18.7 \%) \\
1(6.2 \%) \\
3(18.7 \%) \\
7(43.7 \%) \\
3(18.7 \%) \\
3(18.7 \%) \\
2(12.5 \%) \\
2(12.5 \%)\end{array}$ & $\begin{array}{l}26(53.1 \%) \\
22(44.9 \%) \\
6(12.2 \%) \\
8(16.3 \%) \\
9(18.4 \%) \\
31(63.3 \%) \\
21(42.8 \%) \\
24(49.0 \%) \\
25(51.0 \%) \\
5(10.2 \%) \\
7(14.3 \%)\end{array}$ & $\begin{array}{l}0.035 \\
0.819 \\
0.169 \\
0.873 \\
0.443 \\
0.005 \\
0.819 \\
0.066 \\
0.048 \\
0.835 \\
0.812\end{array}$ \\
\hline Signs/symptoms number, $n^{*}$ & $2(1-2)$ & $3(3-5)$ & 0.031 \\
\hline $\begin{array}{c}\text { Severe COVID-19, } n \\
\text { Hospitalization } \\
\text { Oxygen support } \\
\text { Sequelae }\end{array}$ & $\begin{array}{c}0(0.0 \%) \\
0(0.0 \%) \\
3(12.0 \%)\end{array}$ & $\begin{array}{c}2(4.0 \%) \\
2(4.0 \%) \\
11(22.0 \%)\end{array}$ & $\begin{array}{l}0.314 \\
0.314 \\
0.298\end{array}$ \\
\hline COVID-19 length, days * & $10(7-17)$ & $12(7-21)$ & 0.834 \\
\hline $\begin{array}{c}\text { Time since COVID-19 onset to } \\
\text { swab collection, days * }\end{array}$ & $2(1-3)$ & $3(3-6)$ & 0.088 \\
\hline PCR ORF1ab Cycle threshold & $30.3(24.1-35.5)$ & $22.3(19.6-30.6)$ & 0.023 \\
\hline Parameter & $\begin{array}{l}\text { Totally Vaccinated } \\
\text { HCWs }(n=10)\end{array}$ & $\begin{array}{l}\text { Controls } \\
(n=20)\end{array}$ & $p$ \\
\hline Age, years & $49(37-58)$ & $49(37-58)$ & 1.00 \\
\hline Male, $n$ & $2(20.0 \%)$ & $4(20.0 \%)$ & 1.00 \\
\hline Caucasian, $n$ & $10(100 \%)$ & $18(90.0 \%)$ & 0.540 \\
\hline Comorbidities/subject, $n$ & $0(0-1)$ & $0(0-1)$ & 1.00 \\
\hline Asymptomatic infections, $n$ & $4(40.0 \%)$ & $2(10.0 \%)$ & 0.057 \\
\hline $\begin{array}{c}\text { Signs and Symptoms, } n * \\
\text { Fever } \\
\text { Cough } \\
\text { Rhinorrhea } \\
\text { Pharyngitis } \\
\text { Dyspnea } \\
\text { O/G dysfunction } \\
\text { Headache } \\
\text { Arthromyalgia } \\
\text { Asthenia/Malaise } \\
\text { Nausea/Vomiting } \\
\text { Diarrhea }\end{array}$ & $\begin{array}{c}1(16.7 \%) \\
3(50.0 \%) \\
2(33.3 \%) \\
2(33.3 \%) \\
0(0.0 \%) \\
0(0.0 \%) \\
4(66.7 \%) \\
0(0.0 \%) \\
1(16.7 \%) \\
0(0.0 \%) \\
0(0.0 \%)\end{array}$ & $\begin{array}{c}14(77.8 \%) \\
6(33.3 \%) \\
2(11.1 \%) \\
3(16.7 \%) \\
3(16.7 \%) \\
11(61.1 \%) \\
8(44.4 \%) \\
12(66.7 \%) \\
13(72.2 \%) \\
3(16.7 \%) \\
4(22.2 \%)\end{array}$ & $\begin{array}{l}0.028 \\
0.808 \\
0.527 \\
0.772 \\
0.546 \\
0.016 \\
0.637 \\
0.014 \\
0.050 \\
0.546 \\
0.539\end{array}$ \\
\hline
\end{tabular}


Table 1. Cont.

\begin{tabular}{cccc}
\hline Parameter & $\begin{array}{c}\text { Totally Vaccinated } \\
\text { HCWs }(\boldsymbol{n = 1 0 )}\end{array}$ & $\begin{array}{c}\text { Controls } \\
(\boldsymbol{n}=\mathbf{2 0})\end{array}$ & $p$ \\
\hline Signs/symptoms number, $n$ * & $2(2-3)$ & $4(3-6)$ & 0.014 \\
\hline $\begin{array}{c}\text { Severe COVID-19, } n \\
\text { Hospitalization }\end{array}$ & $0(0.0 \%)$ & $1(5.0 \%)$ & 0.846 \\
Oxygen support & $0(0.0 \%)$ & $1(5.0 \%)$ & 0.846 \\
Sequelae & $0(0.0 \%)$ & $4(20.0 \%)$ & 0.272 \\
\hline COVID-19 length, days * & $5(3-6)$ & $9(7-14)$ & 0.028 \\
\hline $\begin{array}{c}\text { Time since COVID-19 onset to } \\
\text { swab collection, days * }\end{array}$ & $3(3-4)$ & $3(1-5)$ & 0.98 \\
\hline PCR ORF1ab Cycle threshold & $35.0(31.3-35.9)$ & $22.5(18.2-30.6)$ & 0.020 \\
\hline
\end{tabular}

* In symptomatic subjects only. Legend: HCWs, health care workers; $\mathrm{O} / \mathrm{G}$ dysfunction, olfactory and/or gustatory dysfunction; COVID-19, novel coronavirus disease 2019; PCR, polymerase chain reaction.

SARS-CoV-2 positive NPs incidence for PHCWs and THCWs in the study period was 367 cases /100,000 and 169 cases/100,000 persons compared to 2699 cases/100,000 residents in Piedmont in the same temporal span (estimate based on the national surveillance data [15]), resulting in an unadjusted incident relative risk of $0.13(0.12-0.15, p<0.0001)$ and $0.06(0.05-0.07, p<0.0001)$, respectively.

As controls, 110 age/sex-matched unvaccinated individuals were randomly selected. No case nor control had concurrent diseases or was taking drugs with potentiality to interfere with immune response to vaccine or infection. Reasons for testing among controls were presenting COVID-19 compatible signs and symptoms in $80.9 \%$ and being contact of a positive case in 19.1\%. Ct values were available for 93/110 (84.5\%) controls and 47/55 $(85.4 \%)$ cases.

As shown in Supplementary Table S2, no clinical nor virological difference was observed in COVID-19 cases between fHCWs and controls. On the contrary, PHCWs showed higher prevalence of asymptomatic infections, and higher $\mathrm{Ct}$ values at diagnosis compared to matched controls (Table 1; Figures 1 and 2). The time elapsing from COVID-19 onset to swab collection was not different between PHCWs and controls (median 2 versus 3 days, $p=0.088$; Table 1). Symptomatic PHCWs presented less signs and symptoms, and a pattern of COVID-19 featured by a lower prevalence of complaints related to systemic illness (fever, asthenia, and malaise).

No difference was found in comorbidity prevalence, COVID-19-related sequelae, nor in hospitalization and oxygen support requirements between PHCWs or THCWS and controls (Table 1$)$; nevertheless, among the $6(3.6 \%)$ patients requiring hospitalization and oxygen support, 5 were controls and 1 belonged to fHCWs. No subject died among cases and controls.

THCWs showed higher prevalence of asymptomatic infections, and higher Ct values at diagnosis compared to controls (Table 1; Figures 1 and 2). The time elapsing from COVID19 onset to swab collection was not different between THCWs and controls (median 3 versus 3 days, $p=0.98$; Table 1 ). Symptomatic THCWs presented less signs and symptoms, a pattern of COVID-19 with lower prevalence of complaints related to systemic illness (fever, arthromyalgia, asthenia, and malaise), and a shorter duration of signs and symptoms length (median 5 versus 9 days, $p=0.028$; Table 1), which inversely correlated with the time elapsed from first vaccine dose to COVID-19 onset (rho $-0.832, p=0.040$; Figure 3). This correlation was not observed among symptomatic PHCWs (rho 0.251, $p=0.348$ ). 


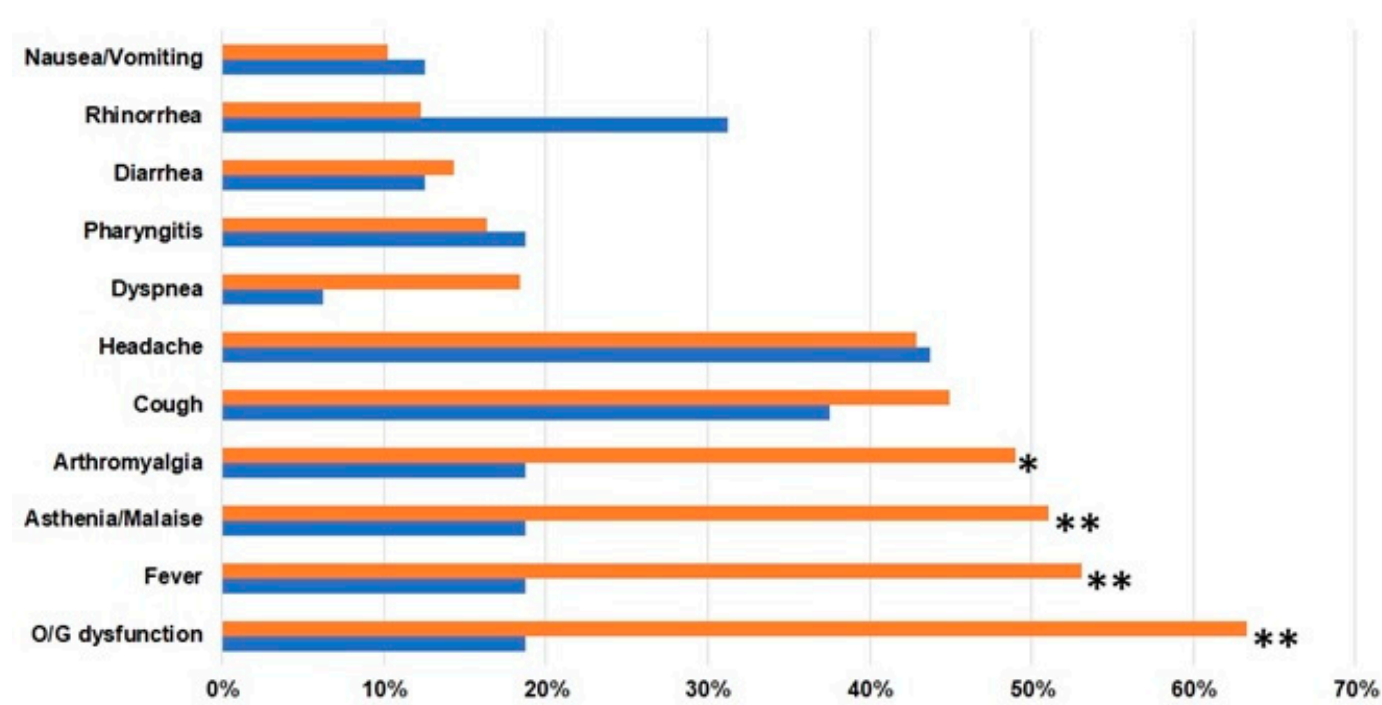

Unvaccinated matched symptomatic controls $(n=49) \quad$ Partially vaccinated symptomatic HCWs $(n=16)$

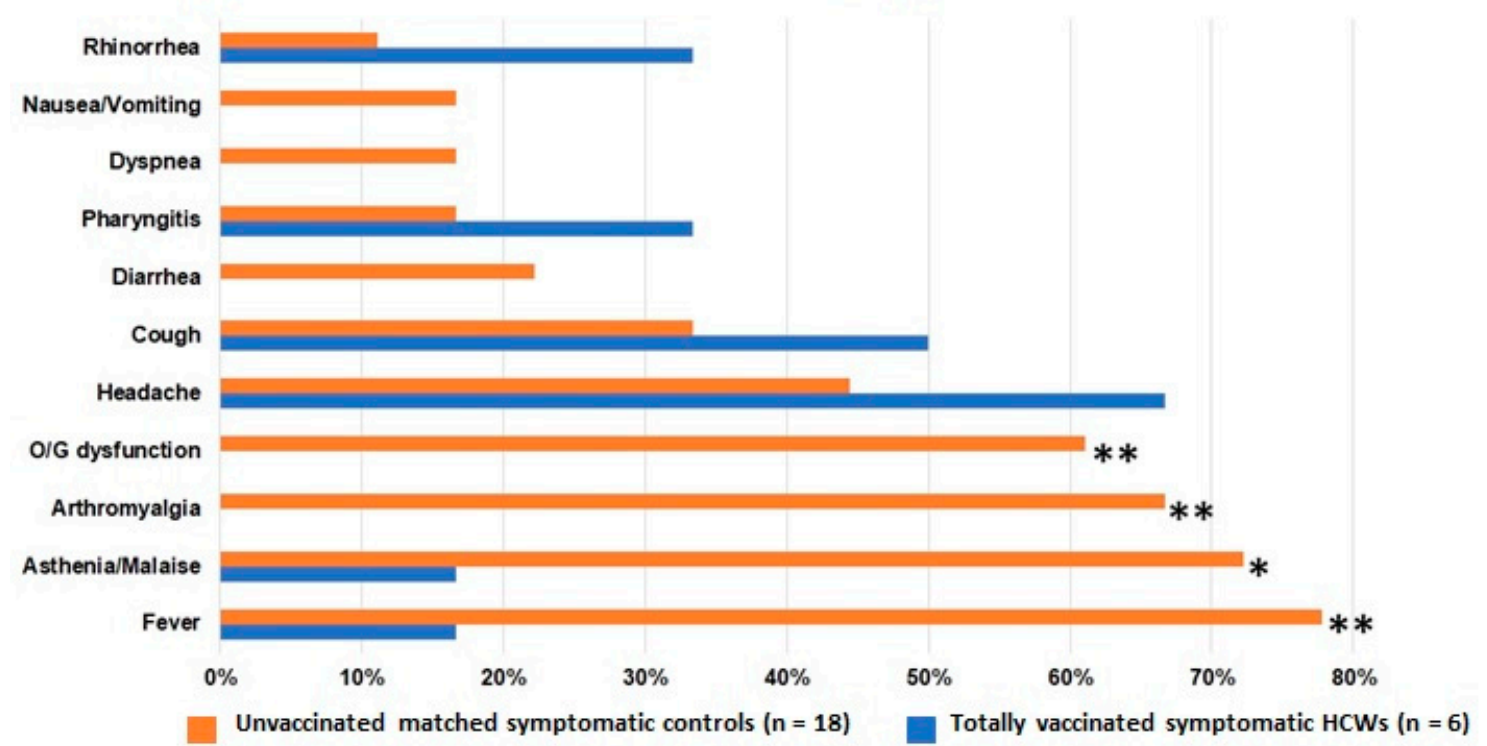

Figure 1. Comparison of COVID-19-related signs and symptoms between partially and totally vaccinated symptomatic health care workers and corresponding unvaccinated age- and sex-matched controls. ${ }^{* *} p$ value $<0.05$; ${ }^{*}$ trend for statistical significance. Legend: HCWs, health care workers; O/G dysfunction, olfactory and/or gustatory dysfunction. 


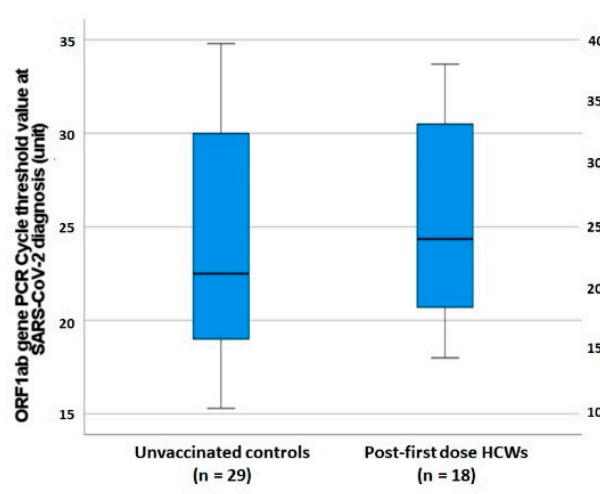

(a)

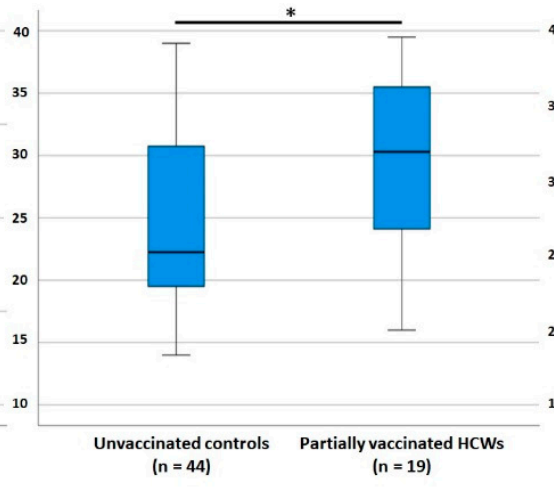

(b)

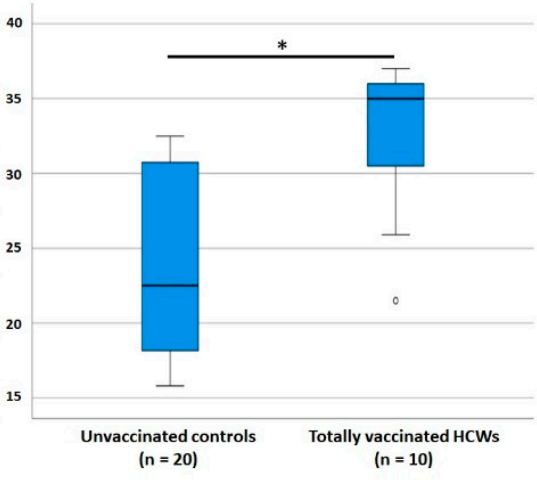

(c)

Figure 2. Comparison of SARS-CoV-2 PCR cycle threshold values across the study population. Comparison of PCR cycle Table 2. diagnosis between corresponding unvaccinated sex- and age-matched controls and post-first dose vaccinated health care workers (a), partially vaccinated health care workers $(\mathbf{b})$, and totally vaccinated health care workers (c). Legend: HCWs, health care workers, PCR, polymerase chain reaction. * Significant differences $(p<0.05)$ were highlighted.

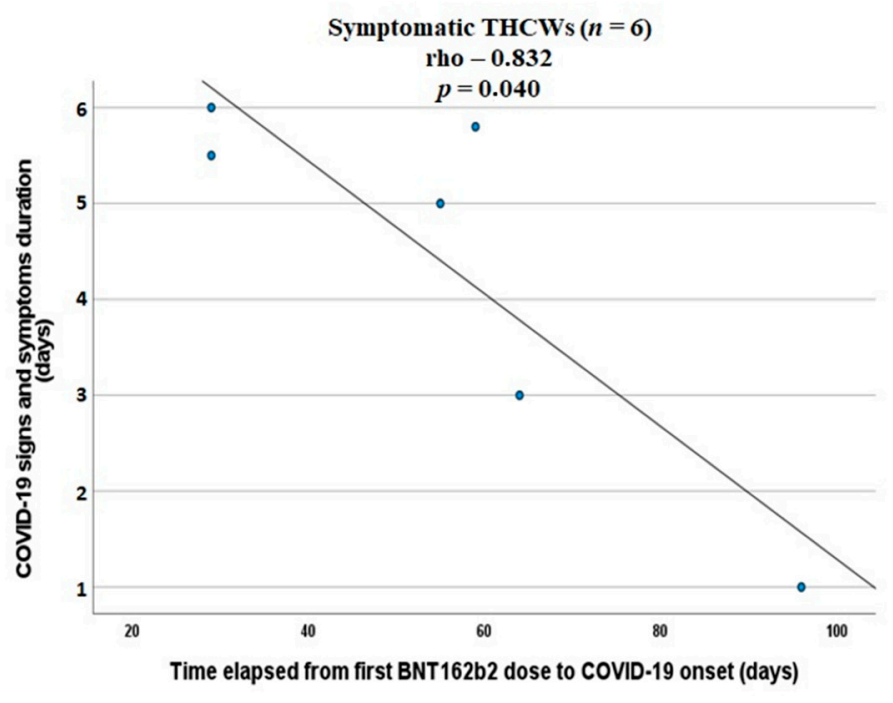

(a)

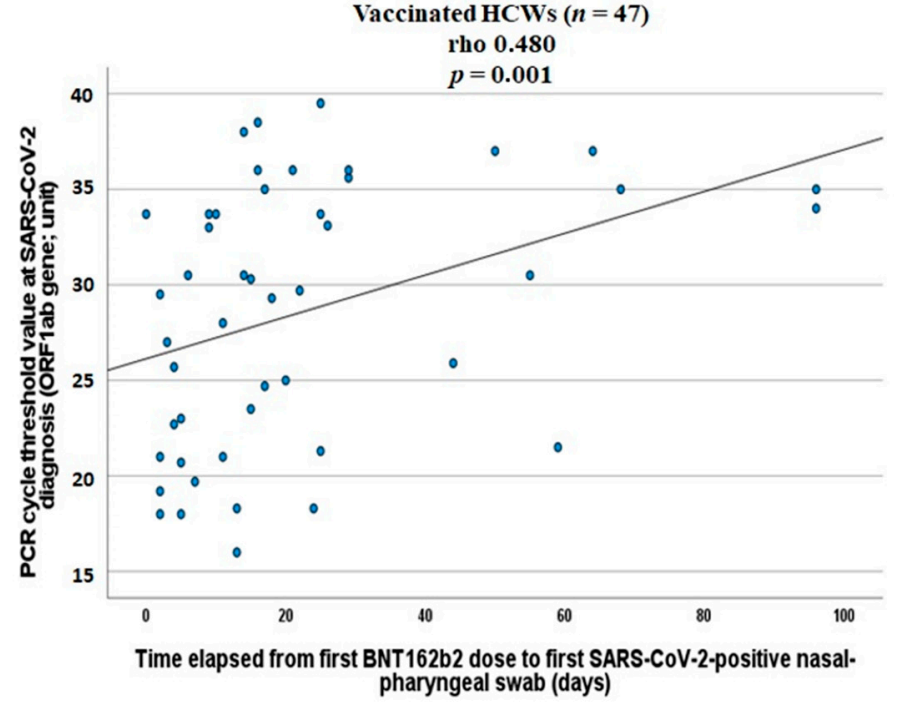

(b)

Figure 3. Correlations between COVID-19-related clinical and virological features in regard to the time from first BNT162b2 vaccine dose. (a) Spearman's correlation between the duration of COVID-19-related signs and symptoms and the time elapsed from first BNT162b2 dose to COVID-19 onset in symptomatic totally vaccinated health care workers. (b) Spearman's correlation between SARS-CoV-2 PCR cycle threshold values at diagnosis and the time elapsed from first BNT162b2 dose to first positive nasal-pharyngeal swab in all vaccinated health care workers.

We observed a significant trend in increasing median $\mathrm{Ct}$ values at diagnosis: from the lowest of the whole unvaccinated controls sample (22.5 (19.5-30.5)) to the highest of THCWs (35.0 (31.3-35.9), $p=0.003$; all the median Ct values per group are reported in Table 1 and Supplementary Table S2). A significant difference in Ct values was maintained even when comparing within the three groups of vaccinated HCWs only $(p=0.036)$. Among symptomatic infections, $\mathrm{Ct}$ values at diagnosis significantly and linearly increased by 3.81 (1.67-5.96) unit according to the vaccination status (unvaccinated controls plus post-first dose cases as reference) after adjusting for other relevant variables such as age, sex, number of comorbidities, and time between COVID-19 onset to swab collection (F 3.6, $p=0.001$; Table 2). Accordingly, among all vaccinated HCWs, the Ct value at the diagnosis increased with increasing time from the first dose to SARS-CoV-2 detection (rho $=0.480$, $p=0.001$; Figure 3). 
Table 2. Linear regression model for the association of SARS-CoV-2 PCR cycle threshold values at diagnosis and vaccination status among symptomatic infections $(n=121)$.

\begin{tabular}{ccc}
\hline Variable & B (95\% CI) & $p$ \\
\hline $\begin{array}{c}\text { Vaccination status (reference: unvaccinated } \\
\text { controls + post-first dose) }\end{array}$ & $3.81(1.67-5.96)$ & 0.001 \\
\hline Age & $-0.006(-0.089-0.76)$ & 0.883 \\
\hline Sex (reference: female) & $-1.64(-4.64-1.37)$ & 0.282 \\
\hline Number of comorbidities & $0.53(-1.03-2.10)$ & 0.500 \\
\hline Days from COVID-19 onset to swab collection & $0.48(0.209-0.754)$ & 0.001 \\
\hline
\end{tabular}

\section{Discussion}

In a setting of healthy young-adult HCWs at high risk of SARS-CoV-2 exposure, during the first three months of the vaccination campaign with BNT162b2 we observed a significant reduction in the odds of SARS-CoV-2 positive swabs compared to the general population, a different pattern of COVID-19 clinical phenotype compared to symptomatic unvaccinated controls, and a reduced prevalence of cases with high potential of contagiousness as soon as 12 days after the first vaccine dose, further decreasing after the second.

In our cohort, BNT162b2 showed $87.0 \%$ and $94.0 \%$ effectiveness in reducing PCRconfirmed infections after 12 and 7 days from the first and second dose, respectively. Other studies in HCWs described reductions in SARS-CoV-2 swab positivity ranging from $42 \%$ to $85 \%$ after a variable time span from the first vaccine dose (7-28 days), with shorter observational period than the current study $[5,8,16,17]$. Angel et al. reported adjusted incident rate ratio of symptomatic infections of 0.11 and 0.03 for partially and fully vaccinated HCWs compared to unvaccinated HCWs and adjusted ratios of asymptomatic infections of 0.64 and 0.14 , respectively [18]. The variability among these findings, including ours, may depend on the adopted temporal cut-offs from vaccination, type of control groups and ethnicity, and by the fact that we could not adjust the incident relative risk for the time-at-risk which may have been different between cases and controls. To date, the majority of studies including ours supports that among young-adult, otherwise healthy subjects at higher risk of repeated exposures to relevant SARS-CoV-2 viral loads, an initial clinical protection is observed as soon as 10-14 days after the first BNT162b2 dose.

Since our cases and controls underwent swab collection due to partially different reasons and frequency, no unbiased conclusion can be drawn about the actual reduction of symptomatic infections following the vaccination in our cohort; nevertheless, this is indeed something that does not require further supporting evidence. Furthermore, as for other observational studies [17-19], the majority of our breakthrough infections were symptomatic $(62.8 \%)$ and the comparison in the clinical phenotype should not have been affected. Symptomatic infections in PHCWs and THCWs were characterized by about half of the amount of signs and symptoms detected in unvaccinated controls, and by a clinical picture mainly resembling that of a mild flu without systemic involvement (cough, rhinorrhea, pharyngitis, and headache only); furthermore, in symptomatic THCWs the disease lasted about half the duration of COVID-19 in unvaccinated symptomatic subjects, where the pattern was more commonly that of a moderate SARS-CoV-2 infection (fever, arthromyalgia, asthenia/malaise, cough, pharyngitis, smell/taste loss).

While the study population (healthy young-adults with a very low burden of comorbidities) reduced the likelihood to detect potential differences in complications of COVID-19, in line with others' findings [19], the lower prevalence of systemic symptoms may reveal a higher barrier to viral dissemination from the initial infection site, which instead might not apply to local respiratory airways, or even a lower ability to trigger inflammation and cytokine-related signs and symptoms (such as fever and malaise). Concerning the olfactory/gustatory dysfunction, its physiopathology is still debated, and our data could fit with the hypothesis that these sensory alterations may associate with an 
indirect central nervous system disturbance by SARS-CoV-2 mediated by inflammatory processes $[20,21]$, potentially reduced by the vaccine-induced specific immunity.

From an epidemiological standpoint, it is not clear to what extent vaccinated individuals who develop asymptomatic and symptomatic infections may represent a reservoir of SARS-CoV-2 transmission as compared to their unvaccinated counterparts [22]. Indeed, the evidence on viral amount carried by vaccinated but infected subjects is scarce and heterogeneous, with emerging evidence from a recent and large cohort in line with our results [23]. The observed parallel increase in Ct values of new infections with increasing time from first vaccination has been already described [19]. In nursing home residents with asymptomatic SARS-CoV-2 infections, a single BNT162b2 dose was associated with -2.4 mean $\log 10$ lower nasopharyngeal load than detected in absence of vaccination [24]. A significant double-step increase in $\mathrm{Ct}$ values (from not vaccinated to partially vaccinated and from partially to fully vaccinated subjects) has also been already recorded [10,25]. Given that $1 \mathrm{Ct}$ unit difference approximates a factor of 2 in the number of virions/sample [10], the linear increase in $\mathrm{Ct}$ we have observed should determine an about 7-fold decrease in viral load from unvaccinated/fHCWs to PHCWs and again from the latter to THCWs. Considering $\mathrm{Ct}$ as an inverse proxy of viral amount and index of transmission potential [26] as well as the early collection of swab from the onset of symptoms of our cases and controls (median 2 and 3 days, Supplementary Table S1), which reassures on the reliable representativeness of the detected $\mathrm{Ct}$ as one of the highest values of viral amount reached in the study subjects as per viral kinetics trends [27], our finding supports the hypothesis that both symptomatic and asymptomatic infections among vaccinated subjects could represent a limited reservoir for onwards transmission, regardless of symptoms and comorbidities. However, no study has already established an in vivo relationship between $\mathrm{Ct}$ values and infectiousness and several factors related to pre-analytical and analytical issues can affect $\mathrm{Ct}$ results and their interpretation. However, while all laboratories can easily access this parameter, the exact quantification of SARS-CoV-2 RNA from human samples is less extensively available and could be more demanding in terms of costs, undergoing partially the same methodological issues. Further investigations on larger study samples should properly address the potential reduced infectiousness of vaccinated individuals based on either $\mathrm{Ct}$ values or the exact quantification of SARS-CoV-2 RNA to confirm our data.

To the best of our knowledge, this is the second real-life study detailing and comparing the clinical presentations and the potential virological contagiousness of post-vaccination infections with a long observational period and matched controls [10]. Furthermore, $\mathrm{Ct}$ values were rarely comparable in terms of analytical methods and performing laboratories, and they were rarely adjusted for the time from disease onset to swab collection [13].

This is a single-district study and missing $\mathrm{Ct}$ values have reduced the already limited sample size for our virological outcome. Test-seeking behaviors, testing frequency, and the reasons behind swab collection were partially different between cases and controls; these factors may have led to an overestimation of the effect size of vaccination on the prevalence of symptomatic infection in controls and of asymptomatic ones among the cases.

Cases were defined by place of work regardless of residency while controls by place of testing; we could not control for several factors that differed between these two populations which may have affected our outcomes, such as higher levels of specific immunity among controls due to previous undetected SARS-CoV-2 infections, adherence to protective measures, exposure risks, or a differential circulation of SARS-CoV-2 strains. Regarding the last issue, half of the study breakthrough infections (51.4\%) occurred during a period where surveillance data for Italy reported a predominance of SARS-CoV-2 B.1.1.7 variant [28]. Indeed, different results could be observed in populations exposed to different SARS-CoV-2 variants of concern. In regard to a potential different circulation of variants between cases and controls, a relevant number of our cases had likely acquired the infection in the community/home and not in hospital since $45.4 \%$ of HCWs had a concurrent or preceding SARS-CoV-2-positive household case, $12.7 \%$ had a concurrent/preceding positive colleague, and $41.8 \%$ had no known positive contacts. 
As the early amount of viral load has been associated with COVID-19 severity [14], the observed lower early viral amount in the upper respiratory airways associated with the vaccination status as soon as 12 days after the first dose can explain the clinical phenotypes recorded in our cohort and endorses the strategy to maximize initial vaccination coverage extending the interval between BNT162b2 doses in otherwise healthy young-adult populations.

\section{Conclusions}

In conclusion, although these data refer to a post-vaccination follow-up period of few months only, and it has yet to be established how long these correlates of immune protection will apply, it appears likely that vaccination further decreases the risk of PCRconfirmed infections and severe disease by wild-type and B.1.17 SARS-CoV-2 strains. It is also associated with a virologic picture of lesser epidemiologic and contagiousness concerns as soon as 12 days after the first vaccine dose. If a confirmation of a relationship between viral amount (expressed by SARS-CoV-2 RNA or its proxy the PCR Ct value) and transmission potential will be provided, this study adds data endorsing a further mechanism by which vaccination can reduce onward transmission: not only by means of a reduction of the overall infection rate but also through the reduction of infectiousness of breakthrough cases.

Supplementary Materials: The following are available online at https:/ /www.mdpi.com/article/10.339 0/vaccines9121377/s1, Table S1: Demographic, clinical, and virological features of the study population, Table S2: Comparison of demographic, clinical and virological features of post-first dose SARS-CoV-2positive healthcare workers and corresponding age- and sex-matched unvaccinated controls.

Author Contributions: Conceptualization, M.T., G.D.P. and A.C.; data curation, F.V., V.G., E.B., T.E. and A.C.; formal analysis, M.T., V.G., E.B. and A.C.; investigation, M.T., F.V., E.B., S.B. and G.D.P.; methodology, M.T., F.V., V.G., E.B., T.E., S.B., G.D.P. and A.C.; supervision, V.G., T.E., S.B., G.D.P. and A.C.; validation, M.T.; visualization, M.T.; writing—original draft, M.T.; writing—review and editing, M.T., F.V., V.G., E.B., T.E., S.B., G.D.P. and A.C. All authors have read and agreed to the published version of the manuscript.

Funding: This research received no external funding.

Institutional Review Board Statement: The study was conducted according to the guidelines of the Declaration of Helsinki and approved by the Inter-departments Ethics Committee A.O.U. Città della Salute e della Scienza, A.O. Ordine Mauriziano di Torino, and A.S.L. Città di Torino (protocol n.0065839-00304, Sept. 2020).

Informed Consent Statement: Informed consent was obtained from all subjects involved in the study.

Data Availability Statement: The datasets generated during and/or analyzed during the current study are available from the corresponding author on reasonable request.

Acknowledgments: We are grateful to the patients and their family, as well as all the professionals working at ASL Città di Torino, for participating in this study and for having faced these hard times with strength and unity.

Conflicts of Interest: The authors declare no conflict of interest.

\section{References}

1. Polack, F.P.; Thomas, S.J.; Kitchin, N.; Absalon, J.; Gurtman, A.; Lockhart, S.; Perez, J.L.; Pérez Marc, G.; Moreira, E.D.; Zerbini, C.; et al. Safety and Efficacy of the BNT162b2 MRNA COVID-19 Vaccine. N. Engl. J. Med. 2020, 383, 2603-2615. [CrossRef]

2. Chodick, G.; Tene, L.; Patalon, T.; Gazit, S.; Tov, A.B.; Cohen, D.; Muhsen, K. The Effectiveness of the First Dose of BNT162b2 Vaccine in Reducing SARS-CoV-2 Infection 13-24 Days after Immunization: Real-World Evidence. medRxiv 2021. [CrossRef]

3. Dagan, N.; Barda, N.; Kepten, E.; Miron, O.; Perchik, S.; Katz, M.A.; Hernán, M.A.; Lipsitch, M.; Reis, B.; Balicer, R.D. BNT162b2 MRNA COVID-19 Vaccine in a Nationwide Mass Vaccination Setting. N. Engl. J. Med. 2021, 384, 1412-1423. [CrossRef]

4. Keehner, J.; Horton, L.E.; Pfeffer, M.A.; Longhurst, C.A.; Schooley, R.T.; Currier, J.S.; Abeles, S.R.; Torriani, F.J. SARS-CoV-2 Infection after Vaccination in Health Care Workers in California. N. Engl. J. Med. 2021, 384, 1774-1775. [CrossRef] 
5. Thompson, M.G.; Burgess, J.L.; Naleway, A.L.; Tyner, H.L.; Yoon, S.K.; Meece, J.; Olsho, L.E.W.; Caban-Martinez, A.J.; Fowlkes, A.; Lutrick, K.; et al. Interim Estimates of Vaccine Effectiveness of BNT162b2 and MRNA-1273 COVID-19 Vaccines in Preventing SARS-CoV-2 Infection among Health Care Personnel, First Responders, and Other Essential and Frontline Workers-Eight U.S. Locations, December 2020-March 2021. MMWR Morb. Mortal. Wkly. Rep. 2021, 70, 495-500. [CrossRef]

6. Menni, C.; Klaser, K.; May, A.; Polidori, L.; Capdevila, J.; Louca, P.; Sudre, C.H.; Nguyen, L.H.; Drew, D.A.; Merino, J.; et al. Vaccine Side-Effects and SARS-CoV-2 Infection after Vaccination in Users of the COVID Symptom Study App in the UK: A Prospective Observational Study. Lancet Infect. Dis. 2021, 21, 939-949. [CrossRef]

7. Westhölter, D.; Taube, C. SARS-CoV-2 Outbreak in a Long-Term Care Facility after Vaccination with BNT162b2. Clin. Infect. Dis. 2021, 2021, ciab299. [CrossRef] [PubMed]

8. Amit, S.; Regev-Yochay, G.; Afek, A.; Kreiss, Y.; Leshem, E. Early Rate Reductions of SARS-CoV-2 Infection and COVID-19 in BNT162b2 Vaccine Recipients. Lancet 2021, 397, 875-877. [CrossRef]

9. Vitiello, A.; Ferrara, F.; Troiano, V.; La Porta, R. COVID-19 Vaccines and Decreased Transmission of SARS-CoV-2. Inflammopharmacology 2021, 29, 1357-1360. [CrossRef]

10. Levine-Tiefenbrun, M.; Yelin, I.; Katz, R.; Herzel, E.; Golan, Z.; Schreiber, L.; Wolf, T.; Nadler, V.; Ben-Tov, A.; Kuint, J.; et al. Initial Report of Decreased SARS-CoV-2 Viral Load after Inoculation with the BNT162b2 Vaccine. Nat. Med. 2021, 27, 790-792. [CrossRef] [PubMed]

11. Padoan, A.; Dall'Olmo, L.; Della Rocca, F.; Barbaro, F.; Cosma, C.; Basso, D.; Cattelan, A.; Cianci, V.; Plebani, M. Antibody Response to First and Second Dose of BNT162b2 in a Cohort of Characterized Healthcare Workers. Clin. Chim. Acta 2021, 519, 60-63. [CrossRef] [PubMed]

12. Kalimuddin, S.; Tham, C.Y.; Qui, M.; de Alwis, R.; Sim, J.X.; Lim, J.M.; Tan, H.-C.; Syenina, A.; Zhang, S.L.; Le Bert, N.; et al. Early T Cell and Binding Antibody Responses Are Associated with COVID-19 RNA Vaccine Efficacy Onset. Med 2021, 2, 682-688. [CrossRef]

13. Tande, A.J.; Pollock, B.D.; Shah, N.D.; Farrugia, G.; Virk, A.; Swift, M.; Breeher, L.; Binnicker, M.; Berbari, E.F. Impact of the COVID-19 Vaccine on Asymptomatic Infection among Patients Undergoing Pre-Procedural COVID-19 Molecular Screening. Clin. Infect. Dis. 2021, 2021, ciab229. [CrossRef]

14. Trunfio, M.; Venuti, F.; Alladio, F.; Longo, B.M.; Burdino, E.; Cerutti, F.; Ghisetti, V.; Bertucci, R.; Picco, C.; Bonora, S.; et al. Diagnostic SARS-CoV-2 Cycle Threshold Value Predicts Disease Severity, Survival, and Six-Month Sequelae in COVID-19 Symptomatic Patients. Viruses 2021, 13, 281. [CrossRef] [PubMed]

15. Dipartimento della Protezione Civile, Consiglio dei Ministri. COVID-19 ITALIA. Available online: https:/ / opendatadpc.maps arcgis.com/apps/dashboards/b0c68bce2cce478eaac82fe38d4138b1 (accessed on 3 August 2021).

16. Benenson, S.; Oster, Y.; Cohen, M.J.; Nir-Paz, R. BNT162b2 MRNA COVID-19 Vaccine Effectiveness among Health Care Workers. N. Engl. J. Med. 2021, 384, 1775-1777. [CrossRef]

17. Tang, L.; Hijano, D.R.; Gaur, A.H.; Geiger, T.L.; Neufeld, E.J.; Hoffman, J.M.; Hayden, R.T. Asymptomatic and Symptomatic SARS-CoV-2 Infections After BNT162b2 Vaccination in a Routinely Screened Workforce. JAMA 2021, 325, 2500. [CrossRef] [PubMed]

18. Angel, Y.; Spitzer, A.; Henig, O.; Saiag, E.; Sprecher, E.; Padova, H.; Ben-Ami, R. Association between Vaccination with BNT162b2 and Incidence of Symptomatic and Asymptomatic SARS-CoV-2 Infections among Health Care Workers. JAMA 2021, $325,2457$. [CrossRef]

19. Pritchard, E.; Matthews, P.C.; Stoesser, N.; Eyre, D.W.; Gethings, O.; Vihta, K.-D.; Jones, J.; House, T.; VanSteenHouse, H.; Bell, I.; et al. Impact of Vaccination on SARS-CoV-2 Cases in the Community: A Population-Based Study Using the UK's COVID-19 Infection Survey. medRxiv 2021. [CrossRef]

20. Speth, M.M.; Singer-Cornelius, T.; Oberle, M.; Gengler, I.; Brockmeier, S.J.; Sedaghat, A.R. Mood, Anxiety and Olfactory Dysfunction in COVID-19: Evidence of Central Nervous System Involvement? Laryngoscope 2020, 130, 2520-2525. [CrossRef]

21. Edén, A.; Kanberg, N.; Gostner, J.; Fuchs, D.; Hagberg, L.; Andersson, L.M.; Lindh, M.; Price, R.W.; Zetterberg, H.; Gisslén, M. CSF Biomarkers in Patients with COVID-19 and Neurologic Symptoms: A Case Series. Neurology 2021, 12, e294-e300. [CrossRef]

22. Donadio, C.; Rainone, A.; Gouronnec, A.; Belmin, J.; Lafuente-Lafuente, C. Asymptomatic COVID-19 Cases among Older Patients despite BNT162b2 Vaccination: A Case Series in a Geriatric Rehabilitation Ward during an Outbreak. J. Infect. 2021, 83, 119-145. [CrossRef]

23. Singanayagam, A.; Hakki, S.; Dunning, J.; Madon, K.J.; Crone, M.A.; Koycheva, A.; Derqui-Fernandez, N.; Barnett, J.L.; Whitfield, M.G.; Varro, R.; et al. Community transmission and viral load kinetics of the SARS-CoV-2 delta (B.1.617.2) variant in vaccinated and unvaccinated individuals in the UK: A prospective, longitudinal, cohort study. Lancet Infect. Dis. 2021. [CrossRef]

24. McEllistrem, M.C.; Clancy, C.J.; Buehrle, D.J.; Lucas, A.; Decker, B.K. Single Dose of a MRNA SARS-CoV-2 Vaccine Is Associated with Lower Nasopharyngeal Viral Load among Nursing Home Residents with Asymptomatic COVID-19. Clin. Infect. Dis. 2021, 73, e1365-e1367. [CrossRef] [PubMed]

25. Jacobson, K.B.; Pinsky, B.A.; Rath, M.E.M.; Wang, H.; Miller, J.A.; Skhiri, M.; Shepard, J.; Mathew, R.; Lee, G.; Bohman, B.; et al. Post-Vaccination SARS-CoV-2 Infections and Incidence of the B.1.427/B.1.429 Variant among Healthcare Personnel at a Northern California Academic Medical Center. medRxiv 2021. [CrossRef]

26. Rao, S.N.; Manissero, D.; Steele, V.R.; Pareja, J. A Systematic Review of the Clinical Utility of Cycle Threshold Values in the Context of COVID-19. Infect. Dis. Ther. 2020, 9, 573-586. [CrossRef] [PubMed] 
27. He, X.; Lau, E.H.Y.; Wu, P.; Deng, X.; Wang, J.; Hao, X.; Lau, Y.C.; Wong, J.Y.; Guan, Y.; Tan, X.; et al. Temporal Dynamics in Viral Shedding and Transmissibility of COVID-19. Nat. Med. 2020, 26, 672-675. [CrossRef] [PubMed]

28. Lai, A.; Bergna, A.; Menzo, S.; Zehender, G.; Caucci, S.; Ghisetti, V.; Rizzo, F.; Maggi, F.; Cerutti, F.; Giurato, G.; et al. Circulating SARS-CoV-2 Variants in Italy, October 2020-March 2021. Virol. J. 2021, 18, 168. [CrossRef] [PubMed] 